\title{
Anteversión pélvica como causa de dolor lumbar, síndrome patelofemoral y dolores del crecimiento
}

\author{
J. A. Vélez-Patiñoํㅜ, L. M. Ríos-Sánchez², F. L. Ochoa-Jaramillo³ y C. A. Díaz-León ${ }^{4}$ \\ ${ }^{1}$ Grupo de Investigación Movimiento y Salud. Ortopedista, Especialidad Columna. ${ }^{2}$ Fisioterapia. \\ Pedagogía/Magíster en Discapacidad. Grupo de Investigación Movimiento y Salud, Universidad CES. \\ ${ }^{3}$ Epidemiología. Grupo de Investigación Observatorio de la Salud Pública. Universidad CES. Medellín, \\ Colombia. ${ }^{4}$ Ingeniería Informática. Grupo de Investigación $I+D+I$ en Tecnologías de la Información y las \\ Comunicaciones. Universidad EAFIT. Medellín, Colombia
}

Vélez-Patiño JA, Ríos-Sánchez LM, Ochoa-Jaramillo FL, Díaz-León CA. Anteversión pélvica como causa de dolor lumbar, sindrome patelofemoral y dolores del crecimiento. Rev Soc Esp Dolor 2014; 21(2): 75-83.

\begin{abstract}
Introduction: Back pain, patellofemoral syndrome and "growing pains" are increasingly common in children and adolescents, both with incidence and precocity of appearance. In most cases their etiology is unknown.

Objective: The objective of this research was to explore the possible association of these three entities with pelvic anteversion.

Methods: This is a cross-sectional study with case-control analysis, comprising a group of 30 symptomatic children aged between 6 and 17 years (cases) who had consulted for low back pain, "growing pains" or sore knees, and a control group of 30 children with no history of pain in these areas (controls). To measure the position of the pelvis and other biomechanical variables a software programme was developed that quantifies these measurements from reflective markings located on specific anatomical landmarks of each child. Other measurements were obtained through semiological tests and interviews with children and their parents.

Results: Children and adolescents with pain had more pelvic anteversion compared with controls (mean 13.3 and 5.4 degrees, $\mathrm{p}<0.001$ ). They also had less strength in muscles: Lumbar extensor, abdominals, psoas and diaphragm. We found
\end{abstract}

Financiación: Dirección de Investigaciones. Universidad CES. Código del proyecto: 200808 di13.

Agradecimientos: Hospital Pablo Tobón Uribe, Colegio Colombo Británico de Envigado y Universidad CES, todos en Colombia.

Recibido: 20-01-13.

Aceptado: 21-04-13. that psoas is the muscle most responsible for pelvis anteversion in symptomatic group. Compared with children without pain, those with back pain, patellofemoral syndrome or "growing pains" have 4.2 times more frequent poor or regular strength in the column extensor muscles. They also have nearly 18 times as likely to have a pelvic angle greater than 10 degrees compared with no pain children.

Conclusions: Children and adolescents who suffer from back pain, patellofemoral syndrome and "growing pains" have increased pelvic anteversion and lower muscle strength, mainly in the thoracolumbar spine extensors, abdominals, diaphragm and psoas. The study found that the psoas muscle is most responsible for the development of pelvis anteversion. Pelvic anteversion is associated with low back pain, patellofemoral pain syndrome and "growing pains".

Key words: Pelvis. Anteversion. Back pain. Patellofemoral pain syndrome. Children.

\section{RESUMEN}

Introducción: el dolor lumbar, el síndrome patelofemoral y los "dolores del crecimiento" son cada vez más frecuentes en niños y adolescentes, tanto en incidencia como en precocidad de aparición. En la mayoría de los casos su etiología se desconoce.

Objetivo: el objetivo de esta investigación fue explorar la asociación de estas tres entidades con la anteversión pélvica.

Métodos: se realizó un estudio transversal, con análisis de casos y controles, en un grupo de 30 niños con edades entre 6 y 17 años que hubieran consultado por dolor lumbar, "dolores de crecimiento" o dolor en las rodillas (casos), y otro grupo conformado por otros 30 menores sin antecedentes de dolor en dichas regiones (controles). Para la medición de la posición de la pelvis y otras variables biomecánicas se desarrolló un software que cuantifica estas medidas a partir de marcas reflectivas ubicadas sobre puntos anatómicos específicos en cada niño. Otras mediciones fueron obtenidas a través de pruebas semiológicas y entrevistas a los menores y sus padres. 
Resultados: se encontró que los menores con dolor presentaron mayor anteversión de la pelvis comparados con aquellos sin dolor (medias de 13,3 y 5,4 grados, $\mathrm{p}<0,001$ ). Igualmente, tenían menor fuerza en los músculos extensores de columna lumbar, abdominales, psoas y diafragma. Encontramos, además, que el psoas es el músculo con mayor responsabilidad en la anteversión de la pelvis en los niños del grupo sintomático. En un menor con dolor lumbar, síndrome patelofemoral o "dolores del crecimiento" fue 4,2 veces más frecuente la presencia de fuerza deficiente o regular de los músculos extensores de columna que en los menores sin dolor. Igualmente, tuvieron casi 18 veces la probabilidad de tener un ángulo pélvico mayor de 10 grados comparado con aquellos menores sin dolor.

Conclusión: los niños y adolescentes que sufren dolor lumbar, síndrome patelofemoral y "dolores de crecimiento" presentan mayor anteversión de la pelvis y menor fuerza muscular, principalmente en los extensores de columna toracolumbar, abdominales, diafragma y psoas. El estudio encontró que el músculo psoas es el que tiene mayor responsabilidad en el desarrollo de anteversión de la pelvis. La anteversión de la pelvis se asocia con dolor lumbar, síndrome patelofemoral y "dolores de crecimiento".

Palabras clave: Pelvis. Anteversión. Dolor lumbar. Síndrome patelofemoral. Dolores del crecimiento. Niños.

\section{INTRODUCCIÓN}

El dolor lumbar (DL), el síndrome patelofemoral (SPF) y los "dolores del crecimiento" (DC) son alteraciones que van en aumento, tanto en frecuencia como en precocidad de aparición, que afectan la calidad de vida y generan altos costos de atención en salud para la economía de muchos países (1-3).

El DL se refiere a un dolor de etiología multifactorial localizado en la parte inferior de la espalda. En el $90 \%$ de los niños y adolescentes no se identifica su etiología. Se ha encontrado relación entre el dolor lumbar y algunos factores, aunque sin establecer una clara asociación de causalidad $(4,5)$. No se ha demostrado la efectividad de la mayoría de los tratamientos para el DL, aunque el $90 \%$ de los casos de lumbalgia pueden ser manejados de forma conservadora mediante distintas alternativas terapéuticas como tratamientos farmacológicos $(6,7)$, procedimientos percutáneos (8), actividad física dirigida y cambios en los hábitos posturales (9); mientras que los procedimientos invasivos se reservan para aquellos casos que no responden al tratamiento conservador (10).

El SPF es un dolor en la parte anterior de las rodillas que se presenta en adolescentes y adultos jóvenes, también de etiología desconocida (11). Afecta al $20 \%$ de la población escolar, aunque su frecuencia puede ser mucho mayor debido al subregistro y la confusión con artralgias inespecíficas, tendinitis, bursitis, meniscopatías y "dolores del crecimien- to". Es más común en mujeres entre los 10 y 20 años de edad y hay genu valgo en el $50 \%$ de los pacientes $(11,12)$.

Una investigación reciente refiere que un protocolo de fisioterapia intensiva y supervisada es mejor que la conducta expectante en el manejo de esta dolencia, mientras que la intervención quirúrgica está indicada en pacientes con luxación recurrente de la patela o dolor grave causado por mala alineación, demostrada por radiografía (13).

Los denominados "dolores de crecimiento" se localizan en la cara anteromedial de las piernas. Se presentan en niños sanos, generalmente al final del día, con periodicidad e intensidad variables, y llegan a alterar sus actividades normales (14). Afectan a alrededor del $37 \%$ de los niños entre los 4 y 6 años y pueden aparecer entre los 2 y 8 años de edad. Tampoco hay completa claridad respecto a su etiología, pero se ha descrito que hasta un $50 \%$ de estos niños presentan acortamiento de los músculos isquiotibiales (14). Tampoco existe un tratamiento específico para el DC, sin embargo, los mejores resultados se obtienen con fisioterapia orientada al estiramiento y al fortalecimiento muscular de las extremidades inferiores (14).

Las entidades descritas anteriormente comparten características comunes: afectación de la población infantil, elevada frecuencia, etiología desconocida y tratamientos inespecíficos de moderada eficacia.

En la experiencia clínica de dos de los autores en el manejo del dolor lumbar se encuentra que muchos pacientes adolescentes reportan entre sus antecedentes personales haber presentado dolor en las rodillas o en las extremidades inferiores durante la infancia, y además poseen ciertas alteraciones biomecánicas (principalmente anteversión de la pelvis, pero también hiperlordosis lumbar, rótulas convergentes o mal alineamiento de las extremidades inferiores), lo que hace plantear la hipótesis de que existe asociación entre dichos dolores en la infancia con la presentación del dolor lumbar en la adolescencia y la edad adulta debido a un mismo origen biomecánico, particularmente por la anteversión pélvica, lo que motivó la presente investigación. El objetivo de esta investigación fue explorar la asociación del dolor lumbar, los dolores de crecimiento y el síndrome patelofemoral con la anteversión pélvica.

\section{MÉTODOS}

Se trata de un estudio de tipo transversal con análisis posterior de tipo casos y controles. Para realizar esta investigación fueron identificados aquellos menores con edades comprendidas entre los 6 y los 17 años que hubieran asistido a la consulta médica de pediatría, ortopedia, fisiatría o reumatología en el Hospital Pablo Tobón Uribe de la ciudad de Medellín (Colombia) durante el periodo 2002-2010 y cuyo motivo de consulta en la historia clínica fuera dolor lumbar, en las rodillas o en las piernas y se les hubiera 
descartado cualquier alteración que fuera la causa de su dolor (neoplasia, artropatías, infecciones y demás causas orgánicas), es decir, sin causa específica de su dolor. Fueron denominados como "casos".

No se realizó cálculo del tamaño de la muestra. Se tomaron, por conveniencia, 30 menores en cada uno de los grupos. Estos menores fueron pareados por edad, sexo y talla con otro grupo de 30 menores sin manifestaciones de dolor en las regiones anatómicas descritas (controles), quienes fueron captados de una institución educativa después de realizarles entrevistas a ellos y a sus padres. Debido a la distinta procedencia de ambos grupos y a que la información se tomó por separado en cada una de las instituciones, no fue posible hacer el cegamiento de los evaluadores.

Ambos grupos fueron evaluados de la misma manera por dos de los investigadores: las variables sociodemográficas fueron registradas mediante entrevista; la fuerza y flexibilidad muscular fueron cuantificadas mediante la escala convencional de 1 a 5 puntos para la fuerza (15) y en grados para la medida de la flexibilidad, de acuerdo con los distintos grupos musculares y la bibliografía al respecto $(16,17)$. Las mediciones fueron realizadas en los músculos: glúteo mayor, extensores de la columna dorsolumbar, recto anterior del abdomen, diafragma, psoas e isquiotibiales, los cuales, por sus inserciones, ejercen una influencia biomecánica sobre la posición de la pelvis.

Las variables biomecánicas fueron medidas a través de un software (Corpomed) desarrollado para esta investigación por uno de los autores (Díaz-León), el cual no ha sido aplicado a otros grupos o en el tiempo, por lo que se trata de una prueba aún no validada. Para ello se colocaron marcadores reflectivos en los siguientes puntos anatómicos: espinas iliacas antero-superior y postero-superior, apófisis espinosas $\mathrm{C}_{7}-\mathrm{S}_{1}$, cóndilo lateral y medial del fémur, centro de la patela, tuberosidad anterior de la tibia y maleolos interno y externo. Posteriormente se tomaban registros fotográficos en posición antero-posterior, lateral y lateral en flexión (Fig. 1) que se enviaban al ordenador y eran procesadas directamente por el software que, de forma automática, detectaba la ubicación de los puntos y registraba las medidas antropométricas y biomecánicas.

La posición de la pelvis en la proyección lateral, medida originalmente en grados con el software Corpomed, fue considerada la variable dependiente del estudio; posteriormente se dicotomizó para el análisis de casos y controles, en mayores (o como exposición) o menores de 10 grados en dicho ángulo.

Se realizó la prueba de Kolmogorov-Smirnov para determinar si se cumplían los preceptos de distribución normal. Se usaron pruebas no paramétricas cuando no se tenía distribución normal de una variable. Se calcularon pruebas t de Student, U de Man Whitney, Fisher o de chi cuadrado, según el tipo de variable.

Para el análisis tipo casos y controles se calcularon los Odds ratio (OR) con sus respectivos intervalos de confianza de $95 \%$ para explorar la asociación entre dolor en cualquiera de las tres localizaciones en relación con el ángulo de la pelvis (mayor o menor de 10 grados) o la fuerza de algunos grupos musculares, que fueron clasificados como fuerza deficiente/regular y adecuada.

Para relacionar la localización del dolor con la posición de la pelvis y algunas variables estudiadas se realizó un análisis de varianza (ANOVA). Para determinar las diferencias en el ángulo de la pelvis en proyección lateral con algunas

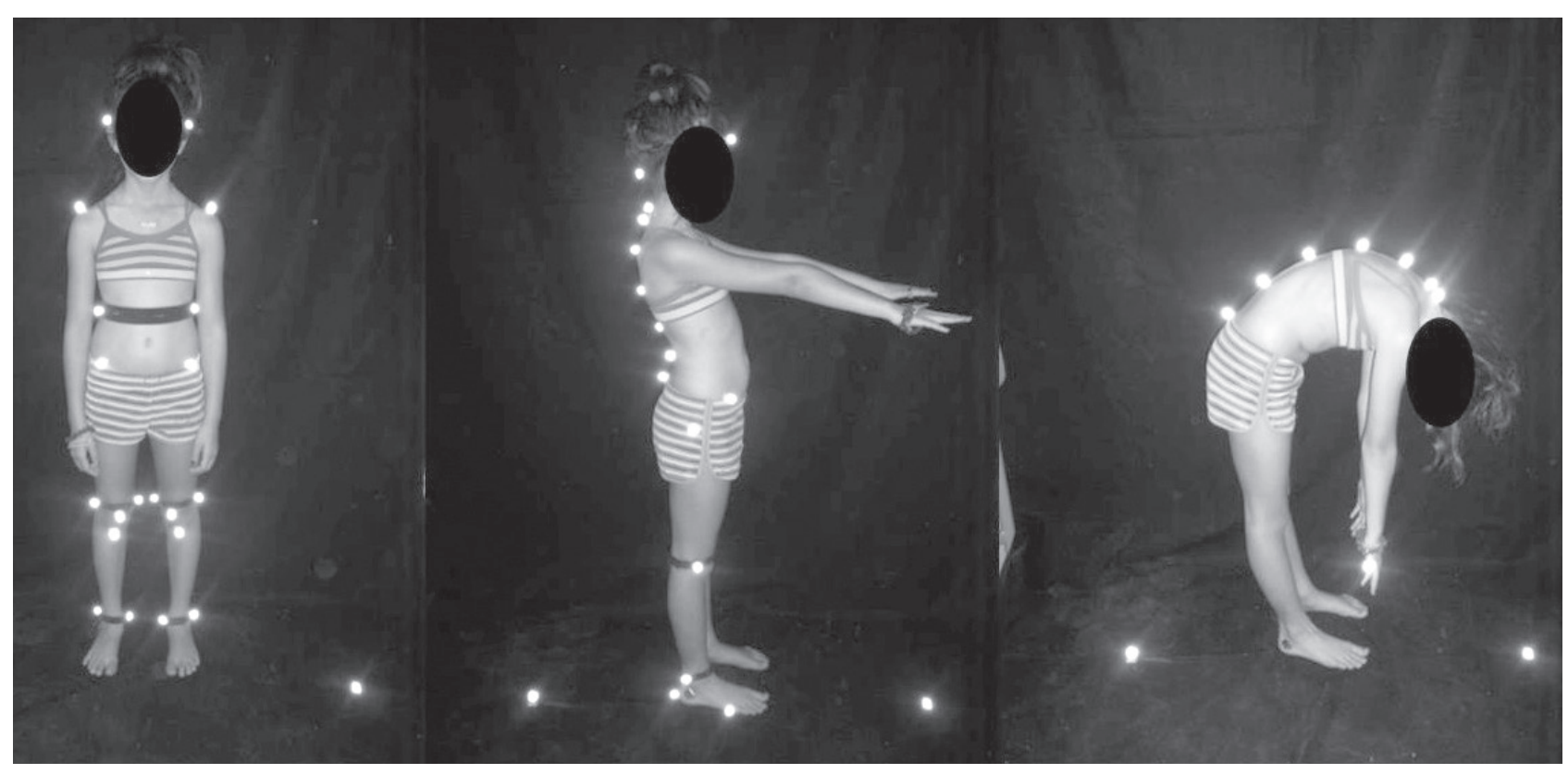

Fig. 1. Sitios de ubicación de los puntos reflectivos. 
variables se calculó la prueba t de Student para las cualitativas con dos categorías, y para aquellas variables con más de dos categorías se calcularon ANOVAS. Para estimar la correlación entre el ángulo de la pelvis en proyección lateral y la flexibilidad de los músculos psoas e isquiotibiales se calculó el coeficiente de correlación de Spearman.

En todos los casos se consideró estadísticamente significativo un valor de $\mathrm{p}$ menor de 0,05 y el intervalo de confianza desde las estimaciones de los OR se obtuvieron con una confianza de $95 \%$.

El estudio fue aprobado por los Comités de Ética de la Universidad CES, del Hospital Pablo Tobón Uribe, y por la Junta Directiva del Colegio Colombo Británico de Medellín. Para todos los casos fue tomado el consentimiento informado de los padres o responsables del menor. Se siguieron los protocolos establecidos en el hospital para acceder a los datos de las historias clínicas de los pacientes. Se obtuvo autorización de los padres o responsables del menor para la toma de las fotografías, las cuales se realizaron siempre en compañía de estos últimos.

\section{RESULTADOS}

En total fueron evaluados 60 menores, 30 en cada uno de los grupos. No se encontraron diferencias en la comparación de los ángulos varo y valgo derecho e izquierdo y en la lordosis lumbar de ambos grupos. Por el contrario, en el ángulo de la pelvis en la proyección lateral todos los menores tuvieron valores mayores de 0 , aunque fue mayor en el grupo de los menores con dolor (media de 13,3 grados y de 5,4 para el grupo de menores sin dolor) $(\mathrm{p}<0,001)$ (Tabla I).
En la fuerza de los músculos extensores de columna se observó un mayor porcentaje de niños con valores de 4 y 5 en el grupo sin dolor ( $56 \%$ y $20 \%$, respectivamente, $\mathrm{p}=$ $0,025)$. En la fuerza del diafragma, un $94 \%$ del grupo sin dolor y un $57 \%$ del grupo de menores con dolor presentaron una fuerza de $5(\mathrm{p}=0,004)$. En la flexibilidad del psoas el $10 \%$ de los menores sin dolor presentó acortamiento, mientras que en el grupo con dolor el $63 \%$ lo presentó $(\mathrm{p}=0,001)$ (Tabla II).

Se encontró correlación significativa, moderada y directamente proporcional entre la flexibilidad del psoas y el ángulo de la pelvis, es decir, que a medida que aumentan los grados de acortamiento del músculo psoas se encuentra mayor anteversión de la pelvis (coeficiente de correlación de Spearman 0,53; $\mathrm{p}=0,000)$. Se encontró correlación estadística entre el ángulo de la pelvis con la fuerza del recto anterior del abdomen, lo que indica que a medida que aumenta la fuerza de este músculo disminuye la anteversión pélvica (Tabla III).

Se cuantificó la asociación entre tener o no dolor (denominados para este análisis como casos y controles) y la exposición (fuerza muscular) en ambos grupos, encontrando una asociación significativa para los músculos recto del abdomen y extensores de columna. Esto quiere decir, por ejemplo, que en un menor con DL, SPF o DC es 4,23 veces más frecuente la presencia de fuerza deficiente o regular de los músculos extensores de columna que en los menores sin dolor (Tabla IV).

Finalmente, se exploró la asociación entre el dolor, en cualquiera de sus tres lugares de presentación, con respecto al ángulo de la pelvis en proyección lateral. Para ello, y a juicio de los investigadores, para el análisis de casos y

TABLA I. CARACTERÍSTICAS DEMOGRÁFICAS, BIOMECÁNICAS Y CLÍNICAS

\begin{tabular}{lccc}
\hline & $\begin{array}{c}\text { Con dolor } \\
n=30\end{array}$ & $\begin{array}{c}\text { Sin dolor } \\
n=30\end{array}$ & Valor $p$ \\
\hline Edad (años) & 11,9 & 11,8 & 0,800 \\
Sexo (\% hombres) & 54 & 54 & 1 \\
Talla (cm)* & $149,6 \pm 16,5$ & $152,3 \pm 14,1$ & 0,510 \\
Peso (kg)* & $42,7 \pm 11,5$ & $43,6 \pm 12,7$ & 0,775 \\
Ángulo de la pelvis proyección lateral (grados)* & $13,3 \pm 6$ & $5,4 \pm 5,2$ & 0,000 \\
Ángulo varo valgo derecho proyección anterior (grados)* & $8 \pm 3,7$ & $7,2 \pm 3,1$ & 0,397 \\
Ángulo varo valgo izquierdo proyección anterior (grados)* & $9 \pm 3,4$ & $8,3 \pm 3,2$ & 0,432 \\
Ángulo lordosis lumbar proyección lateral (grados)* & $175,3 \pm 11,4$ & $172,3 \pm 10,2$ & 0,287 \\
Flexibilidad isquiotibiales* & $34,8 \pm 21,3$ & $26,5 \pm 15$ & 0,085 \\
\hline
\end{tabular}

*Prueba t de Student. $n=30$ para cada grupo. 
TABLA II. COMPARACIÓN DE VARIABLES CLÍNICAS EN AMBOS GRUPOS

\begin{tabular}{|c|c|c|c|c|}
\hline \multirow{3}{*}{ Variable } & \multirow{3}{*}{ Categoría } & \multicolumn{2}{|c|}{ Grupo } & \multirow{3}{*}{ Valor p } \\
\hline & & $\begin{array}{c}\text { Con } \\
\text { dolor }\end{array}$ & $\begin{array}{c}\text { Sin } \\
\text { dolor }\end{array}$ & \\
\hline & & $\%$ & $\%$ & \\
\hline \multirow{5}{*}{ Fuerza de los extensores de columna ${ }^{a}$} & 1 & 3 & 0 & \multirow{5}{*}{0,025} \\
\hline & 2 & 37 & 10 & \\
\hline & 3 & 40 & 33 & \\
\hline & 4 & 17 & 43 & \\
\hline & 5 & 3 & 13 & \\
\hline \multirow{5}{*}{ Fuerza glúteo mayor ${ }^{\mathrm{a}}$} & 1 & - & - & \multirow{5}{*}{0,076} \\
\hline & 2 & 10 & 0 & \\
\hline & 3 & 17 & 13 & \\
\hline & 4 & 53 & 40 & \\
\hline & 5 & 20 & 47 & \\
\hline \multirow{5}{*}{ Fuerza recto anterior abdomen ${ }^{a}$} & 1 & - & - & \multirow{5}{*}{0,105} \\
\hline & 2 & 10 & 0 & \\
\hline & 3 & 27 & 13 & \\
\hline & 4 & 33 & 57 & \\
\hline & 5 & 30 & 30 & \\
\hline \multirow{5}{*}{ Fuerza del psoas ${ }^{\mathrm{a}}$} & 1 & - & - & \multirow{5}{*}{0,363} \\
\hline & 2 & - & - & \\
\hline & 3 & 30 & 33 & \\
\hline & 4 & 47 & 30 & \\
\hline & 5 & 23 & 37 & \\
\hline \multirow{3}{*}{ Fuerza del diafragma $^{a}$} & 3 & 10 & 3 & \multirow{3}{*}{0,004} \\
\hline & 4 & 33 & 3 & \\
\hline & 5 & 57 & 94 & \\
\hline \multirow{2}{*}{ Flexibilidad recto anterior del cuádriceps $\mathrm{s}^{\mathrm{a}, \mathrm{b}}$} & Sin acortamiento & 97 & 100 & \multirow{2}{*}{0,500} \\
\hline & Con acortamiento & 3 & 0 & \\
\hline \multirow{7}{*}{ Flexibilidad del psoas ${ }^{\mathrm{a}}$} & 0 & 37 & 90 & \multirow{7}{*}{0,001} \\
\hline & 4 & 3 & 0 & \\
\hline & 5 & 20 & 0 & \\
\hline & 8 & 0 & 3 & \\
\hline & 10 & 27 & 7 & \\
\hline & 15 & 10 & 0 & \\
\hline & 20 & 3 & 0 & \\
\hline \multirow{3}{*}{ Patrón respiratorio ${ }^{c}$} & Costal superior & 10 & 7 & \multirow{3}{*}{0,549} \\
\hline & Abdominal & 90 & 90 & \\
\hline & Mixto & 0 & 3 & \\
\hline
\end{tabular}

${ }^{a}$ Prueba chi cuadrado o prueba exacta de Fisher. ${ }^{b}$ El acortamiento muscular se refiere a la disminución de la flexibilidad muscular y rango de movilidad articular, mediante goniometría. La ausencia de acortamiento se refiere a una flexibilidad normal con un arco de movilidad adecuado. 'Se tuvo en cuenta la evaluación del patrón respiratorio costal superior (1), el patrón respiratorio abdominal (2) y el patrón respiratorio mixto (3). 
TABLA III. DIFERENCIA DEL ÁNGULO DE LA PELVIS. PROYECCIÓN LATERAL SEGÚN VARIABLES DE FUERZA Y FLEXIBILIDAD MUSCULAR

\begin{tabular}{|c|c|c|c|c|}
\hline \multirow[b]{2}{*}{ Variable } & \multirow[b]{2}{*}{ Categoría } & \multicolumn{2}{|c|}{ Ángulo de la pelvis proyección lateral } & \multirow[b]{2}{*}{ Valor $p$} \\
\hline & & $n$ & $\begin{array}{c}\text { Media } \pm \text { Desviación } \\
\text { estándar }\end{array}$ & \\
\hline \multirow{2}{*}{$\begin{array}{l}\text { Flexibilidad } \\
\text { recto anterior del } \\
\text { cuádriceps }^{\mathrm{a}}\end{array}$} & Sin acortamiento & 59 & $9,23 \pm 6,84$ & \multirow{2}{*}{0,417} \\
\hline & Con acortamiento & 1 & $14,87 \pm \mathrm{NA}$ & \\
\hline \multirow{3}{*}{ Fuerza diafragma $^{\mathrm{b}}$} & 3 & 4 & $12,00 \pm 7,02$ & \multirow{3}{*}{0,180} \\
\hline & 4 & 11 & $12,22 \pm 7,21$ & \\
\hline & 5 & 45 & $8,39 \pm 6,60$ & \\
\hline \multirow{4}{*}{$\begin{array}{l}\text { Fuerza recto anterior } \\
\text { abdomen }^{\mathrm{b}}\end{array}$} & 2 & 3 & $19,16 \pm 2,71$ & \multirow{4}{*}{0,018} \\
\hline & 3 & 12 & $11,88 \pm 4,89$ & \\
\hline & 4 & 27 & $8,06 \pm 6,95$ & \\
\hline & 5 & 18 & $7,89 \pm 6,71$ & \\
\hline \multirow{5}{*}{$\begin{array}{l}\text { Extensores de } \\
\text { columna }^{\text {b }}\end{array}$} & 1 & 1 & $13,06 \pm \mathrm{SD}$ & \multirow{5}{*}{0,150} \\
\hline & 2 & 14 & $11,96 \pm 7,69$ & \\
\hline & 3 & 22 & $9,51 \pm 6,05$ & \\
\hline & 4 & 18 & $8,57 \pm 6,47$ & \\
\hline & 5 & 5 & $3,17 \pm 6,79$ & \\
\hline
\end{tabular}

${ }^{a}$ Prueba t de Student; ${ }^{b}$ ANOVA.

TABLA IV. FUERZA DE ASOCIACIÓN (OR) ENTRE LOS GRUPOS DE CASOS Y CONTROLES RELACIONADOS CON LA FUERZA MUSCULAR Y EL ÁNGULO DE LA PELVIS

\begin{tabular}{|c|c|c|c|c|c|c|}
\hline \multirow[b]{2}{*}{ Variable } & \multirow[b]{2}{*}{ Categoría } & \multicolumn{2}{|c|}{ Grupo } & \multirow[b]{2}{*}{$C h i^{2 a}$} & \multirow[b]{2}{*}{ Valor $p$} & \multirow{2}{*}{$\begin{array}{c}O R \\
(I C 95 \%)\end{array}$} \\
\hline & & $\begin{array}{c}\text { Con dolor } \\
n=30\end{array}$ & $\begin{array}{c}\begin{array}{c}\text { Sin dolor } \\
n=30\end{array} \\
\text { n }\end{array}$ & & & \\
\hline \multirow{2}{*}{$\begin{array}{l}\text { Fuerza extensores } \\
\text { de columna }\end{array}$} & Fuerza deficiente-regular & 24 & 13 & \multirow{2}{*}{8,53} & \multirow{2}{*}{0,03} & \multirow{2}{*}{$\begin{array}{c}5,2 \\
(1,66-16,5)\end{array}$} \\
\hline & Fuerza adecuada & 6 & 17 & & & \\
\hline \multirow{2}{*}{ Fuerza glúteo mayor } & Fuerza deficiente-regular & 8 & 4 & \multirow{2}{*}{1,67} & \multirow{2}{*}{0,197} & \multirow{2}{*}{$\begin{array}{c}2,36 \\
(0,63-8,91)\end{array}$} \\
\hline & Fuerza adecuada & 22 & 26 & & & \\
\hline \multirow{2}{*}{$\begin{array}{l}\text { Fuerza recto anterior } \\
\text { abdomen }\end{array}$} & Fuerza deficiente-regular & 11 & 4 & \multirow{2}{*}{4,36} & \multirow{2}{*}{0,04} & \multirow{2}{*}{$\begin{array}{c}3,7 \\
(1,04-13,65)\end{array}$} \\
\hline & Fuerza adecuada & 19 & 26 & & & \\
\hline \multirow{2}{*}{ Fuerza diafragma } & Fuerza deficiente-regular & 3 & 1 & \multirow{2}{*}{1,071} & \multirow{2}{*}{0,30} & \multirow{2}{*}{$\begin{array}{c}3,22 \\
(0,32-32,9)\end{array}$} \\
\hline & Fuerza adecuada & 27 & 29 & & & \\
\hline \multirow{2}{*}{ Ángulo de la pelvis } & $\geq 10$ & 22 & 4 & \multirow{2}{*}{21,991} & \multirow{2}{*}{0,000} & \multirow{2}{*}{$\begin{array}{c}17,9 \\
(4,7-67,4)\end{array}$} \\
\hline & $<10$ & 8 & 26 & & & \\
\hline
\end{tabular}

a Prueba chi cuadrado o prueba exacta de Fisher; IC $95 \%$ : intervalo de confianza del $95 \%$.

controles, se consideró esta última variable como si fuera dicotómica, de manera que aquellos menores con ángulo de proyección de la pelvis mayor o igual a 10 grados fueron comparados con los menores con menos de 10 grados en el ángulo pélvico. El OR obtenido fue 17,9 (IC $95 \%$ 4,7467,4), es decir, que uno de los menores evaluados y con dolor tiene casi 18 veces la probabilidad de tener un ángulo pélvico mayor de 10 grados que uno sin dolor. 


\section{DISCUSIÓN}

Los resultados de esta investigación permiten aproximarse a la confirmación de la hipótesis de que existe asociación entre la anteversión de la pelvis con DL, SPF y DC en niños y jóvenes. A pesar de que existen numerosos estudios acerca del dolor lumbar en niños y adolescentes, no se encontraron informes relacionados con el dolor y alteraciones en la posición de la pelvis. Sólo un estudio ha demostrado cómo influye la postura de niños y jóvenes en diferentes actividades en el dolor lumbar no específico, aunque no hace mención acerca de cambios estructurales o biomecánicos que pudieran presentarse en la pelvis (18).

Es necesario mencionar que la decisión del punto de corte para el ángulo de la pelvis en posición lateral fue arbitraria pues, de un lado no existe soporte bibliográfico para determinarlo, y del otro porque todos los niños (con y sin dolor) tuvieron valores del ángulo pélvico mayores de cero, por lo que si se hubieran partido los grupos en menores de 0 y mayores de 0 el primero hubiera quedado $\sin$ datos.

En los menores con dolor se encontró menor fuerza muscular en los extensores de la columna toracolumbar, menor flexibilidad del psoas (mayor acortamiento) y mayor anteversión pélvica. Ello podría significar que la anteversión pélvica en los pacientes sintomáticos de dolor lumbar, patelofemoral o de crecimiento podría depender fundamentalmente del acortamiento del psoas, favorecido por una debilidad de los músculos extensores de la columna toracolumbar, tradicionalmente reconocidos como posturales y antigravitacionales. Consideramos que este acortamiento es causado por una flexión permanente de la cadera, iniciada precozmente desde la infancia.

O'Sullivan y Beales (19) resaltan la importancia biomecánica del diafragma, reconociendo su papel en las alteraciones lumbopélvicas. En este estudio se encontró disminución de la fuerza muscular del diafragma en los menores con dolor. Tal situación podría explicarse por el hecho de que un diafragma débil y que no se contrae adecuadamente durante la respiración torácica permanece elongado y tracciona las vértebras lumbares hacia adelante, comportándose como un músculo lordotizante, que contribuye a la anteversión pélvica (20).

Se encontró asociación entre la fuerza del recto anterior del abdomen con la anteversión pélvica: a menor fuerza del recto anterior del abdomen hay mayor anteversión pélvica, lo cual se explica por las inserciones del recto anterior del abdomen en la zona de la sínfisis púbica, que lo convierten en un extensor de la pelvis (21).

En cuanto a la flexibilidad del recto anterior del cuádriceps y de los isquiotibiales, no hubo diferencia entre los grupos en relación con el ángulo de la pelvis. Este hallazgo es de resaltar, ya que, tradicionalmente, ambos grupos musculares han sido importantes en la recuperación y tratamiento del paciente con lumbalgia (9), lo cual contrasta con nuestros hallazgos y deberán ser evaluados en futuras investigaciones.

En resumen, si bien se trata de un estudio exploratorio y de una muestra por conveniencia que limitan su validez externa, los resultados de este estudio apuntan a la asociación entre la anteversión pélvica y la presentación del dolor en los tres sitios descritos.

Al respecto de la anteversión pélvica, que consideramos como la variable principal en esta investigación, no conocemos trabajos recientes que hayan explorado su relación con la presencia de dolor, aunque ya en 1986 Hungria-Filho planteaba la importancia de la musculatura postural y de los músculos que se insertan en la pelvis (22).

Basándose en estos resultados y en los trabajos del Dr. Hungria-Filho se propone una hipótesis acerca del origen del dolor lumbar, el síndrome patelofemoral y los dolores del crecimiento que se explicará a continuación.

Desde los primeros días de vida se van originando alteraciones posturales por la manera en que habitualmente se sostiene a los niños cuando están de brazos: en flexión de cadera y rodillas (23). Más tarde, durante la niñez, con las actividades escolares y en la vida cotidiana de la edad adulta se asumen constantemente posiciones en flexión de cadera y rodillas, puesto que permanecemos mucha parte del tiempo sentados. En otras palabras, en el mundo moderno, y desde una perspectiva biomecánica, el ser humano casi siempre está flexionado en todas sus articulaciones, lo que propicia un desequilibrio entre la musculatura flexora y extensora (22).

La contracción permanente del psoas (principal músculo flexor de la cadera) sin la correspondiente acción de sus antagonistas (recto abdominal, glúteo mayor e isquiotibiales) tracciona la pelvis hacia delante y arrastra consigo la columna lumbar, aumentando entonces su curvatura (hiperlordosis) y la presión en las estructuras posteriores de la columna lumbar, principalmente en las articulaciones facetarias, lo que origina el síndrome facetario, que sería una de las causas de lumbalgia $(22,24)$.

Se han considerado las vertebras $\mathrm{L}_{4} \mathrm{y} \mathrm{L}_{5}$ como las más móviles de la región lumbar, lo que podría no ser cierto, debido a unos potentes ligamentos iliolumbares estabilizadores de la región lumbosacra extendidos oblicuamente desde la cresta iliaca hasta $\mathrm{L}_{4} \mathrm{y} \mathrm{L}_{5}$, constituyéndose un verdadero cimiento de la columna vertebral formado por dichas vértebras, el sacro y las crestas iliacas, con una movilidad restringida $(21,25)$.

Desafortunadamente, estas condiciones biomecánicas se han ido modificando como consecuencia de la manera incorrecta de flexionar el tronco, la cual debería ser realizada aproximadamente en un $80 \%$ en la articulación de la cadera y solamente un $20 \%$ en la columna toracolumbar $(24,26,27)$. La permanente flexión de las caderas va produciendo acortamientos musculares y contracturas capsulares y ligamentarias alrededor de las articulaciones coxofemo- 
rales, por lo que se invierten dichos valores y el $80 \%$ de la flexión se realiza entonces en la columna, lo cual va forzando la movilidad de $\mathrm{L}_{4}$ y L produciendo inicialmente hipermovilidad manifestada como molestias ocasionales, luego se va inestabilizando todo el segmento y aparece entonces la lumbalgia.

Esta demanda permanente va originando cambios estructurales progresivos en $\mathrm{L}_{4}-\mathrm{L}_{5}$ y $\mathrm{S}_{1}$ : artrosis facetarias, degeneración de los discos, hernias, inestabilidades y listesis $(21,22)$. Se conoce que este segmento es el más frecuentemente comprometido en la lumbalgia y también es allí donde se realiza el mayor número de intervenciones quirúrgicas $(1,6)$.

Desde el nacimiento, y de forma espontánea, la respiración tiene un patrón abdominal: durante la inspiración el diafragma, que se inserta en las vértebras lumbares, se contrae y desciende. Durante la espiración sucede lo contrario: el diafragma se relaja, asciende al tórax y se retrae el abdomen (20). Sin embargo, a medida que el niño crece va cambiando su respiración a un patrón torácico. Entonces el diafragma permanece ascendido tensionando las vértebras lumbares hacia adelante y produciendo una mayor curvatura lumbar, lo que genera otra posible causa de lumbalgia (20).

Cuando la pelvis está en anteversión empuja hacia abajo originando fuerzas compresivas en las extremidades inferiores que, en última instancia, se transmiten a las rodillas. Aquí los ligamentos son más fuertes en el lado externo que en el interno, por lo cual las rodillas tienden a desviarse en valgo, la extremidad pierde su alineación y la rótula no se moviliza libremente dentro del surco patelofemoral (12). En consecuencia, cada vez que se desplaza golpea contra las paredes del surco aumentando la fricción y produciendo desgaste en la superficie articular de la rótula. Esto sería la hiperpresión patelar que clínicamente se manifiesta como dolor en la parte anterior de las rodillas (22).

Este mismo vector de fuerza descendente, causado por la anteversión pélvica, también ocasiona un conflicto de fuerzas compresivas en toda la extensión de las extremidades inferiores, por lo que quizás el continuo estrés originado en la resistencia que opone la microarquitectura ósea genere una fatiga del hueso dando lugar a los llamados "dolores del crecimiento" (28). Al respecto, una investigación reciente sugiere que la disminución en la resistencia ósea puede ser un factor contribuyente en la patogénesis de los dolores del crecimiento (29).

Los hallazgos anteriores nos llevan a proponer la hipótesis de que la enfermedad lumbar del adulto estaría precedida desde la infancia por el dolor lumbar, el síndrome patelofemoral y los dolores del crecimiento que pudiesen tener un mismo origen biomecánico: la tendencia de la pelvis hacia la anteversión, lo cual se podría detectar y tratar precozmente. Son necesarios nuevos estudios que analicen tal afirmación.

\section{CORRESPONDENCIA:}

Jorge Augusto Vélez-Patiño

Calle 7 \# 39-107

Médical, Consultorio 1405

Medellín-Colombia.

e-mail: saludaxial@gmail.com

\section{BIBLIOGRAFÍA}

1. Jones GT, Macfarlane GJ. Epidemiology of low back pain in children and adolescents. Arch Dis Child 2005;90:312-6.

2. Luo X, Pietrobon R, Sun SX, Liu GG, Hey L. Estimates and patterns of direct health care expenditures among individuals with back pain in the United States. Spine 2003;29:79-86.

3. Maetzel A, Li L. The economic burden of low back pain: A review of studies published between 1996 and 2001. Best Pract Res ClinRheumatol 2002;16:23-30.

4. Kaspiris A, Grivas TB, Zafiropoulou C, Vasiliadis E, Tsadira O. Nonspecific low back pain during childhood: A retrospective epidemiological study of risk factors. J Clin Rheumatol 2010;16(2):55-60.

5. Roth-Isigkeit A, Schwarzenberger J, Baumeier W, Meier T, Lindig M, Schmucker P. Risk factors for back pain in children and adolescents. Schmerz 2005;19(6):535-43.

6. Tulder MW van, Touray T, Furlan AD, Solway S, Bouter LM. Miorrelajantes para el tratamiento del dolor lumbar inespecífico (Revisión Cochrane traducida). En: La Biblioteca Cochrane Plus, 2008 Número 4. Oxford: Update Software Ltd. Disponible en: http://www.update-software.com (Traducida de The Cochrane Library, 2008 Issue 3. Chichester, UK: John Wiley \& Sons, Ltd.).

7. Deshpande A, Furlan A, Mailis-Gagnon A, Atlas S, Turk D. Opiáceos para el dolor lumbar crónico (Revisión Cochrane traducida). En: La Biblioteca Cochrane Plus, 2008 Número 4. Oxford: Update Software Ltd. Disponible en: http://www. update-software.com (Traducida de The Cochrane Library, 2008 Issue 3. Chichester, UK: John Wiley \& Sons, Ltd.).

8. Staal JB, de Bie R, de Vet HC, Hildebrandt J, Nelemans P. Injection therapy for subacute and chronic low-back pain. Cochrane Database Syst Rev 2008;(3):CD001824.

9. Heymans MW, van Tulder MW, Esmail R, Bombardier C, Koes BW. Escuelas de espalda para el dolor lumbar inespecífico (Revisión Cochrane traducida). En: La Biblioteca Cochrane Plus, 2008 Número 4. Oxford: Update Software Ltd. Disponible en: http://www.update-software.com (Traducida de The Cochrane Library, 2008 Issue 3. Chichester, UK: John Wiley \& Sons, Ltd.).

10. Ibrahim T, Tleyjeh IM, Gabbar O. Surgical versus non-surgical treatment of chronic low back pain: A meta-analysis of randomised trials. Int Orthop 2008;32(1):107-13. Epub 2006 Nov 21.

11. Earl JE, Vetter CS. Patellofemoral pain. Phys Med RehabilClin N Am 2007; 18:439-58.

12. Tállay A, Kynsburg A, Tóth S, Szendi P, Pavlik A, Balogh E, et al. Prevalence of patellofemoral pain syndrome. Evaluation of the role of biomechanical malalignments and the role of sport activity. Orv Hetil 2004;145(41):2093-101.

13. Van Linschoten R, Koëter S. Patellofemoral pain: Physiotherapy and surgery. Ned TijdschrGeneeskd 2010;154:A822.

14. Vergara E, Perilla R, Álvarez G. Dolor de crecimiento. Revisión de la literatura. Rev Col Ortop Traum 2008;22(3):192-5. 
15. Medical Research Council. Aids to the examination of the peripheral nervous system, Memorandum no. 45, Her Majesty's Stationery Office, London, 1981.

16. Norkin CC, White DJ. Goniometría, evaluación de la movilidad articular. $1^{\mathrm{a}}$ ed. Madrid: Marban; 2006.

17. Hislop, Helen J. Daniels-Worthingham's pruebas funcionales musculares: técnicas de exploración manual. Madrid: Marban Libros; 1997.

18. Ramos Pinto R, de S. Guerino C, Bittencourt Consolin D, Violino da Cunha AC. Relação entre lordose lombar e desempenho da musculatura abdominal em alunos de fisioterapia. Acta Fisiátrica 2000;7(3):95-8.

19. O'Sullivan PB, Beales DJ. Changes in pelvic floor and diaphragm kinematics and respiratory patterns in subjects with sacroiliac joint pain following a motor learning intervention: A case series. Man Ther 2007;12(3):209-18.

20. Downey R. Anatomy of the normal diaphragm. Thorac Surg Clin 2011;21(2):273-9.

21. Richard L Broke, Wayne Vogl, Adam W, M Mitchell. Greys Anatomy. Toronto: Ed Elsevier Churchill Livingstone; 2005.
22. Hungria Filho JS. Postura: a primazia da pélvis no seu condicionamento e na correção de seus desvios. Rev Bras Ortop 1986;21:236-42.

23. Bronowski J. El ascenso del hombre. Medellín: Printer Colombiana S. A.; 1.983.

24. Hernandez Corvo R. Morfología Funcional Humana. Sistema Locomotor. Bogotá: Ed. Kinesis; 1998.

25. Leong JC, Luk KD, Chow DH, Woo CW. The biomechanical functions of the iliolumbar ligament in maintaining stability of the lumbosacral junction. Spine 1987;12(7):669-74.

26. Bado JL, Barros PC, Ruggiero A, Navillat M. Síndrome de retracción de isquiotibiales: Apartado de los "Anales de la Facultad de Medicina" - Montevideo 1964;49(1):328-37.

27. Bado JL. Dorso Curvo. Montevideo: Artecolor; 1977.

28. Quintero Esguerra J. Presentación del Libro "Compendio de Ortopedia y Fracturas”. Medicina 2005;27(2).

29. Friedland O, Hashkes PJ, Jaber L, Cohen A, Eliakim A, Wolach B, et al. Decreased bone strength in children with growing pains as measured by quantitative ultrasound. $\mathrm{J}$ Rheumatol 2005; 32:1354-7. 